\title{
PROBLEMAS RELACIONADOS ÀS PENICILINAS NO SETOR DA PEDIATRIA DA FUNDAÇÃO ASSISTENCIAL DA PARAÍBA
}

\section{Penicilin related problems at the pediatric sector of the fundação assistencial da paraíba}

\author{
Daniela A. Vilar ${ }^{1 *}$; Lindomar F. Belém²; Marina S. A. Vilar \\ ${ }^{1}$ Acadêmica do curso de Farmácia da UEPB, UNIVERSIDADE ESTADUAL DA PARAÍBA - \\ UEPB ENDEREÇO: AVENIDA DAS BARAÚNAS Nº 351 \\ ${ }^{2}$ Prof $^{a}$ da disciplina de Farmacotécnica da UEPB \\ ${ }^{3}$ Profa $^{\mathrm{a}}$ da disciplina de Farmacologia da FCM \\ DEPARTAMENTO DE FARMÁCIA - UEPB
}

*Autor para correspondência e-mail:: dani_1011@yahoo.com.br

Recebido em 21/02/2008 - Aceito em 09/09/2008

RESUMO: Em pacientes infantis, o uso de medicamentos leva com freqüência ao surgimento de Problemas Relacionados com Medicamentos (PRM) e exige estratégias de prevenção da morbimortalidade relacionada a esses produtos. Atenção farmacêutica é a provisão responsável da farmacoterapia com o objetivo de alcançar resultados definidos que melhorem a qualidade de vida dos pacientes. A prática da atenção farmacêutica pode reduzir os problemas preveníveis relacionados à farmacoterapia. As penicilinas são antibióticos beta-lactâmicos, utilizadas como primeira escolha para muitas infecções, o que pode desencadear vários problemas aos usuários, sendo um deles os PRMs. Objetivou-se avaliar os PRM às Penicilinas em pacientes hospitalizados no setor da Pediatria da Fundação Assistencial da Paraíba. A pesquisa constituiu-se de trinta e seis pacientes que compuseram um estudo exploratório, do tipo transversal, utilizando um questionário padrão para obtenção de suspeita de Problemas Relacionados às Penicilinas juntamente com a observação dos pacientes durante o período de tratamento. Dos 36 pacientes acompanhados, 17\% apresentaram PRMs Reais, classificando-se em PRM do tipo 6. Quanto às falhas na farmacoterapia, verificou-se a porcentagem de $17 \%$ dos casos para a segurança. A febre constituiu 55\% das reações adversas diagnosticadas. O reduzido número de PRMs detectados assegura a utilização correta das penicilinas na Fundação Assistencial da Paraíba. De uma forma geral, a pesquisa mostrou resultados positivos, evidenciando-se os principais Problemas Relacionados aos Medicamentos que estão relacionados com a Penicilina, abrindo assim espaço para discussões futuras sobre as soluções que possam ser postas em prática, para que se obtenha um tratamento terapêutico mais seguro.

PALAVRAS-CHAVE: Penicilina, Hospital, Pacientes.

ABSTRACT:In children patients, the use of medication frequently leads to the development of drug-related problems (DRP) and demands prevention strategies about morbidity and mortality related to medication. Pharmaceutical care is the responsible provision of drug therapy for the purpose of achieving definite outcomes that improve a patient's quality of life. The model of pharmaceutical care can reduce the burden of preventable drug therapy problems. Penicilines are a beta-lactamics antibiotics, used as the primary choice for many infecctions, which can start many problems to users, one of them being the DRPs. The aim was to evaluate the DRP to the penicilins in pacients allocated at the Pediatric Sector of the "Fundação Assistencial da Paraíba". The research had thirty six patients that participated on an exploratory study, of a transversal type, using a standard questionary to get the Penicilin Drug-Related suspects and also with the observation of patients during the treatment period. From the 36 patients observed, 17\% presented real DRPs, classified as DRP type 6 . As to pharmacotherapy failures, it was verified that $17 \%$ of the cases were for safety. Fever was $55 \%$ of adverse reactions diagnosticated. The few number of Medical Related Problems detected assures the correct usage of penicilins at the "Fundação Assistencial da Paraíba". In general the research showed positive results, identifying the main drug-related problems which are related to Penicilin, opening future discutions on solutions that can be used to obtain a safer therapeutic treatment.

KEYWORDS: Penicilin, Hospital, Patients. 
Vilar, D. A. et al./Revista Eletrônica de Farmácia Vol 5(2), 37-42, 2008.

A Atenção Farmacêutica foi definida pela primeira vez por Hepler e Strand (1999), como a provisão responsável do tratamento farmacológico com o propósito de alcançar resultados concretos que melhorem a qualidade de vida dos pacientes. A mesma envolve o processo pelo qual o farmacêutico participa no desempenho e implementação do surgimento terapêutico do paciente através de um sistema de solução de Problemas Relacionados aos Medicamentos (PRMs), onde a avaliação e monitorização do uso do medicamento são importantes (JARAMILLO, 2003).

PRM é uma questão de Saúde Pública, relacionado ou suspeito de estar associado à farmacoterapia, que interfere ou pode interferir nos resultados terapêuticos e na qualidade de vida do usuário. O PRM é real, quando manifestado, ou potencial na possibilidade de sua ocorrência. Pode ser ocasionado por diferentes causas, tais como: as relacionadas ao sistema de saúde, ao usuário e seus aspectos bio-psico-sociais, aos profissionais de saúde e ao medicamento. A identificação de PRMs segue os princípios de necessidade, efetividade e segurança, próprios da farmacoterapia.

As penicilinas são antibióticos beta-lactâmicos, utilizadas como primeira escolha para muitas infecções, o que pode desencadear vários problemas aos usuários, sendo um deles os PRMs (SILVA, 2002).

Neste sentido, um maior acompanhamento do tratamento farmacológico, na busca de encontrar e documentar PRMs-penicilinas favorece uma melhor capacidade de avaliação da relação benefício/risco, otimizando os resultados da terapêutica e contribuindo para a melhoria da qualidade de vida e adequação do arsenal terapêutico (CIPOLLE et al.,2004).

O presente estudo teve por objetivo avaliar os PRMs-Penicilinas em pacientes hospitalizados no setor da Pediatria da Fundação Assistencial da Paraíba (FAP).

\section{MATERIAL E MÉTODOS}

A pesquisa foi realizada através de um estudo transversal, com a finalidade de avaliar os PRMs Penicilinas, em pacientes hospitalizados no hospital da FAP, em Campina Grande - PB, durante o período estabelecido no cronograma para a coleta dos dados.

A amostra pesquisada foi constituída de 36 pacientes que estavam sob tratamento com Penicilinas, no período de agosto de 2005 a junho de 2006.

Foi utilizado como instrumento de coleta de dados, questionário padrão, elaborado especificamente para a realização do estudo.

A metodologia de seguimento farmacoterapêutico adotada para a Atenção Farmacêutica na FAP é a preconizada pelo Programa Dáder (DÁDER et al., 2002).

Os dados coletados foram inseridos em tabelas, de modo que possibilitaram a realização de uma análise quantitativa, considerando-se os valores relativos e absolutos que justificam a prevalência das respostas. De posse dos resultados, realizou-se uma abordagem qualitativa confrontando os dados obtidos com os dados da literatura. de Saúde.

Este trabalho segue a resolução número 196, de 10 de outubro de 1996, segundo o Conselho Nacional

\section{RESULTADOS E DISCUSSÃO}

Os resultados apresentados são referentes ao período de Agosto de 2005 a Julho de 2006 de seguimento farmacoterapêutico a 36 pacientes sob uso de penicilinas na Ala da Pediatria da FAP. Neste período foram detectados $17 \%$ de PRMs através da sistemática de identificação da metodologia Dáder (DÁDER, et al.,2002) (Figura 1).

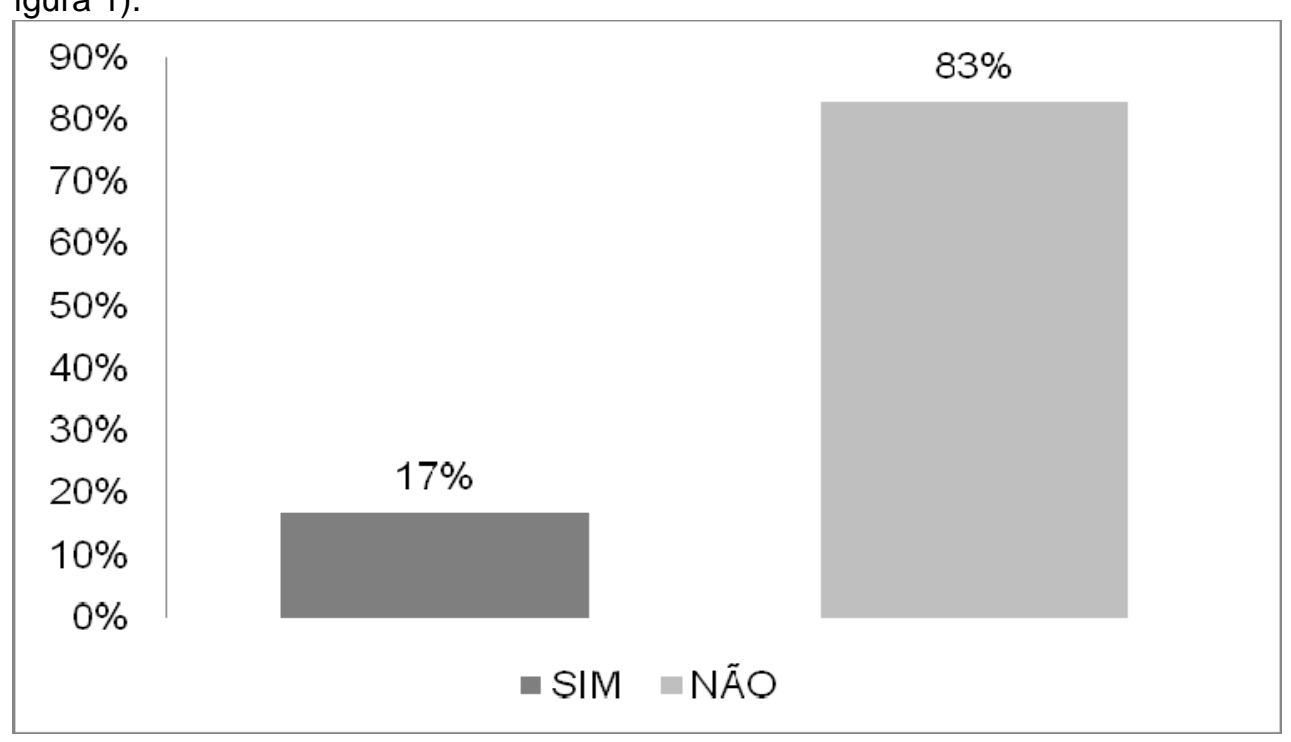


Vilar, D. A. et al./Revista Eletrônica de Farmácia Vol 5(2), 37-42, 2008.

FIGURA 1: Percentual de Problemas Relacionados aos Medicamentos-Penicilinas-acompanhados durante Agosto de 2005 a Julho de 2006.

A utilização de uma sistemática de classificação é uma importante ferramenta na identificação de PRMs, abordando temas, como adesão terapêutica, erros de medicação, automedicação, interações medicamentosas e reações adversas aos medicamentos (RAMs).

A organização e avaliação das informações de cada paciente basearam-se no proposto pelo método Dáder (MACHUCA et al., 2003) e a avaliação de PRM seguiu a classificação do Segundo Consenso de Granada (2002) (Quadro I). A partir de uma análise focada no processo de uso de medicamentos, foram avaliados os riscos de PRM a que os pacientes estavam expostos. Não foram avaliados os resultados clínicos da terapêutica naquele momento.

QUADRO I - Classificação de Problemas Relacionados com Medicamentos (PRM), de acordo com o segundo consenso de Granada (Comité de Consenso, 2002).

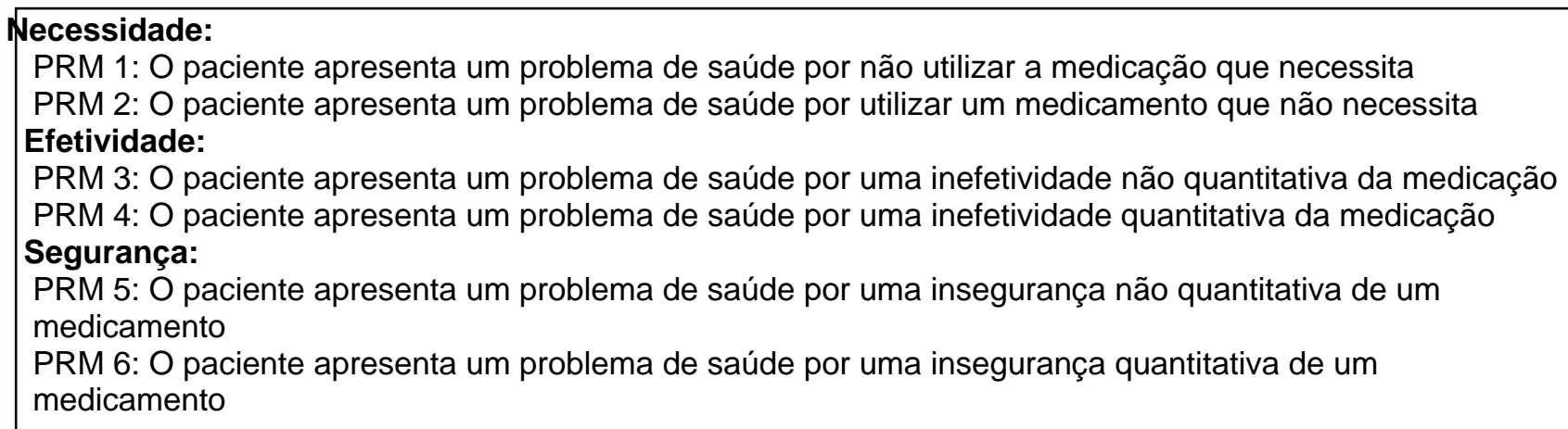

Com a análise dos dados obtidos pode-se observar que dos pacientes acompanhados $70 \%$ eram homens, tendo uma maior ocorrência no mês de março (Tabela 1).

TABELA 1: Perfil dos pacientes acompanhados na Fundação Assistencial da Paraíba - FAP durante os meses do estudo.

\begin{tabular}{lcc}
\hline MESES & HOMENS & MULHERES \\
\hline AGOSTO & 0 & 1 \\
SETEMBRO & 1 & 1 \\
OUTUBRO & 3 & 0 \\
NOVEMBRO & 1 & 1 \\
DEZEMBRO & 2 & 0 \\
JANEIRO & 2 & 0 \\
FEVEREIRO & 2 & 1 \\
MARÇO & 5 & 4 \\
ABRIL & 3 & 1 \\
MAIO & 4 & 0 \\
JULHO & 4 & 2 \\
\hline Total & 25 & 11 \\
\hline
\end{tabular}

De acordo com a classificação de PRMs segundo Dáder et al.,(2002), nesse estudo foram detectados PRMs reais $17 \%$ e PRMs potenciais 83\% (Figura 2). 
Vilar, D. A. et al./Revista Eletrônica de Farmácia Vol 5(2), 37-42, 2008.

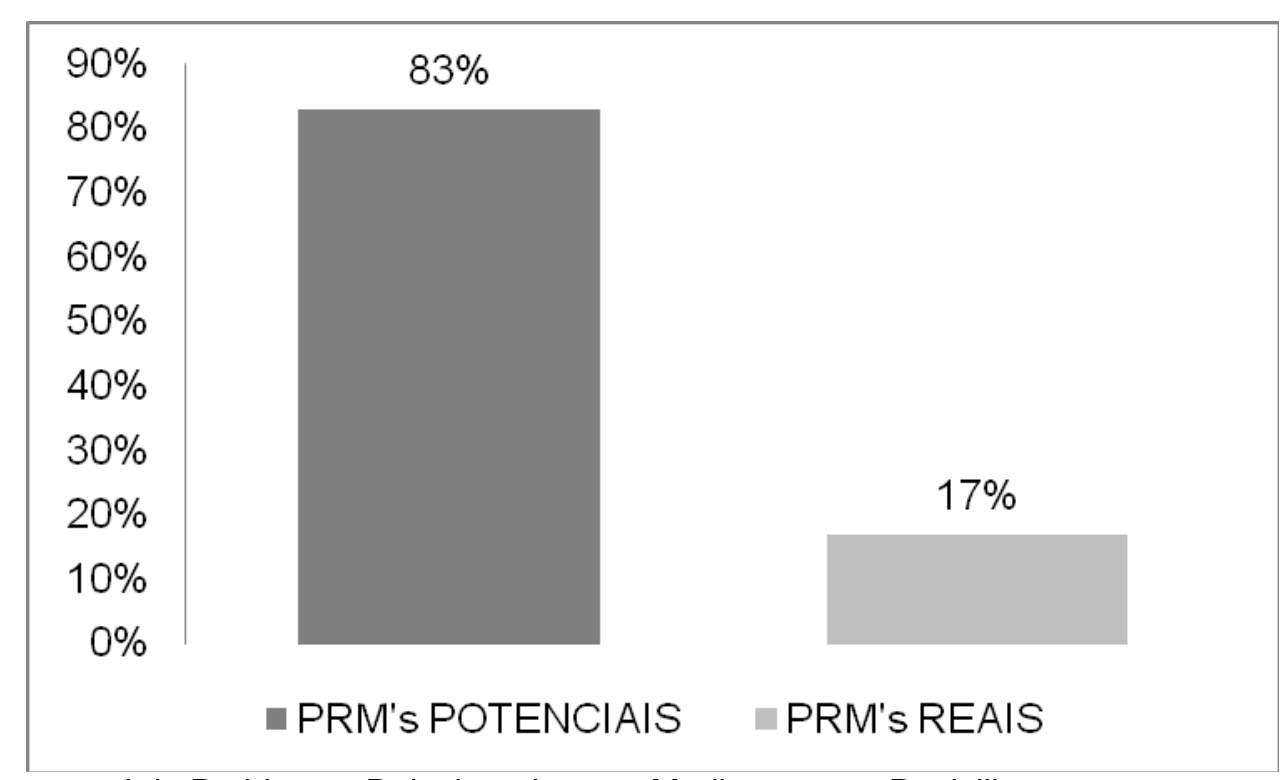

FIGURA 2: Percentual de Problemas Relacionados aos Medicamentos-Penicilinas quanto a sua manifestação.

Na FIGURA 3 observa-se a distribuição dos PRMs baseando-se na classificação de falhas na farmacoterapia: necessidade, efetividade e segurança.

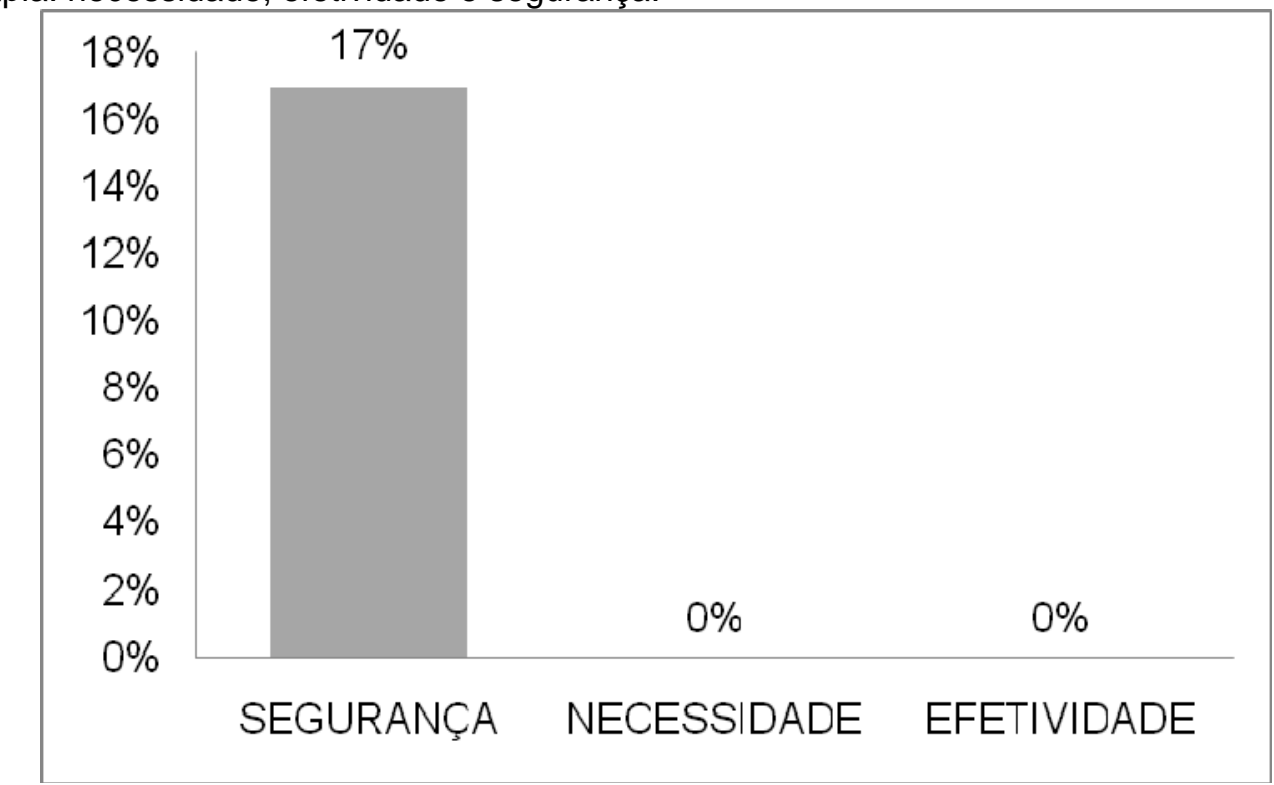

FIGURA 3: Percentual de Problemas Relacionados aos Medicamentos-Penicilinas quanto às falhas na farmacoterapia.

Segundo Strand et al., (1999), de acordo com a classificação em oito tipos de PRM's, foram detectados 6 PRM's classificando-os no PRM tipo 6 (o paciente utiliza um medicamento que lhe causa RAM).

Como o PRM do tipo 6 esta relacionado com a utilização de um medicamento que causa RAM ao paciente, observou-se que dentre as RAMs comprovadas está a febre com 55\% (Figura 4). 
Vilar, D. A. et al./Revista Eletrônica de Farmácia Vol 5(2), 37-42, 2008.

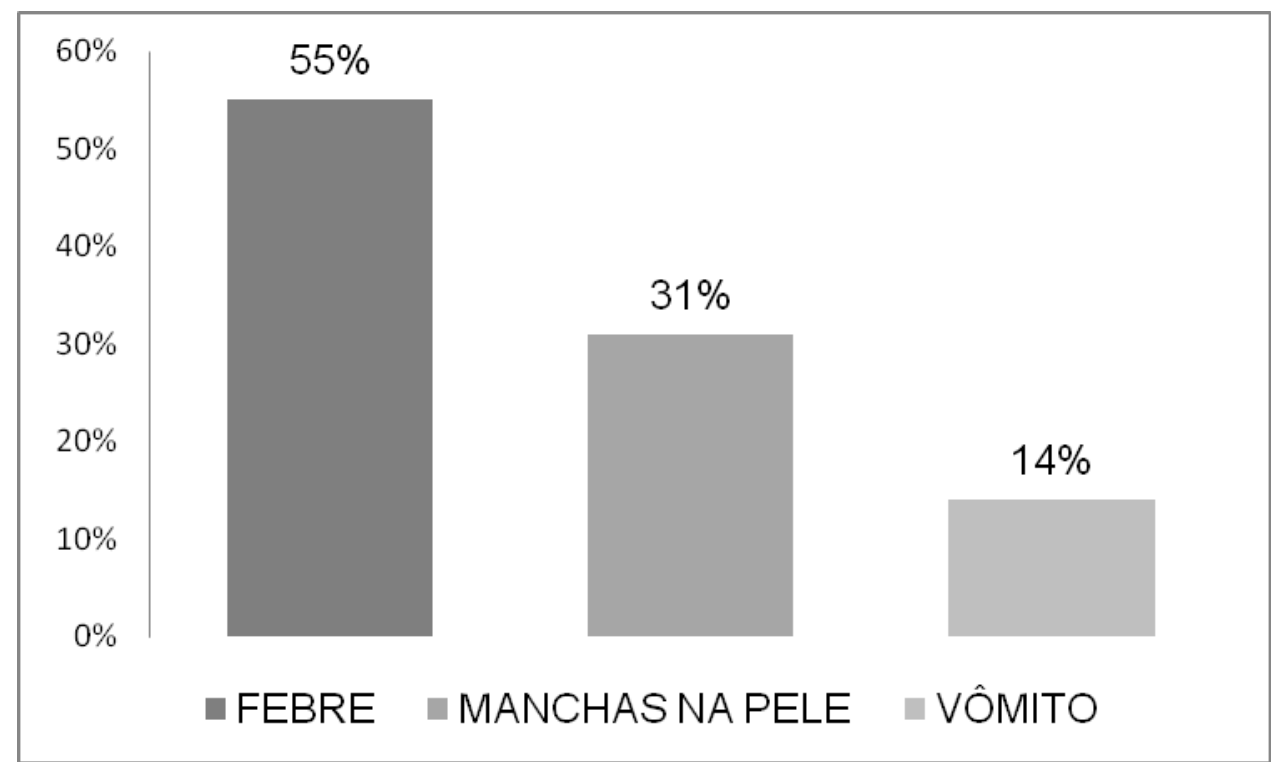

FIGURA 4: Percentual das Reações Adversas as Penicilinas encontradas durante o estudo.

Sendo assim comum à ocorrência de erupções cutâneas e febre; raramente, observa-se um tipo tardio de doença do soro (urticária, febre, edema articular, edema angioneurótico, prurido intenso e distúrbio respiratório) (GOODMAN, 2004).

Neste sentido, um maior acompanhamento do tratamento farmacológico, na busca de encontrar e documentar PRMs-penicilinas favorece uma melhor capacidade de avaliação da relação benefício/risco, otimizando os resultados da terapêutica e contribuindo para a melhoria da qualidade de vida e adequação do arsenal terapêutico.

Contudo a não comprovação de muitos PRMs não indica que o estudo foi realizado de forma incorreta, mas sim que a utilização das penicilinas na pediatria do Hospital da FAP é feita de forma correta.

\section{CONCLUSÕES}

Dos 36 sujeitos assistidos, 6 apresentaram PRM. Contudo a não comprovação de muitos PRMs não indica que o estudo foi realizado de forma incorreta, mas sim que a utilização das penicilinas na pediatria do Hospital da FAP é feita de forma correta, confirmando assim, que o farmacêutico é o profissional qualificado para otimizar a terapia medicamentosa, prevenindo, detectando e corrigindo PRM's, garantindo portanto o sucesso da terapia farmacológica, melhorando a qualidade de vida do paciente e contribuindo para a redução dos custos hospitalares.

\section{REFERÊNCIAS BIBLIOGRÁFICAS}

CIPOLLE, R. J.; STRAND, L. M.; MORLEY, P. C. Pharmaceutical care practice: The clinician's Guide. New York: McGraw-Hill, 2004. 624p.

COMITE DE CONSENSO. Segundo Consenso de Granada sobre Problemas Relacionados aos Medicamentos. Grupo de Investigación em Atención Farmacêutica. Ars Pharmaceutica, 2002.

DÁDER, F. M. J.; FERNANDEZ-LLIMÓS, F.; MARTINEZ ROMERO, F. Programa dader de implantación del seguimiento del tratamiento farmacológico. Ars pharmaceutica, v. 43, p. 175-184, 2002.

GOODMAN, A.G. As Bases Farmacológicas da Terapêutica. Ed. Mc Graw-Hill. Rio de Janeiro,10ª Edição, 2004.

HEPLER, C. D.; STRAND, L. M. Oportunidades y responsabilidades en la atención farmacéutica. Tradución de la Fundación Pharmaceutical Care Espanã. Pharmaceutical Care España, Granada, v.1, p. 35-47, 1999. Título original: Oportunities and responsabilities in the pharmaceutical care.

JARAMILLO, N. M. Uma proposta de consenso para a Atenção Farmacêutica. Pharmacia Brasileira - Mai / Jun. 2003, p 14-19.

MACHUCA, M.; FERNÁNDEZ-LLIMÓS, F.; FAUS, M.J. Método Dáder. Guía de Seguimiento Farmacoterapêutico. Granada: UGR, 2003. 43 p. 
Vilar, D. A. et al./Revista Eletrônica de Farmácia Vol 5(2), 37-42, 2008.

SILVA, P. Farmacologia. $6^{\circ}$ ed., Rio de Janeiro: Editora Guanabara Koogan, 2003.

STRAND L. M. et al. Problemas relacionados con el medicamento: sua estructura y sua función. Pharmaceutical Care España, 1999. 